\title{
Potential Prognostic Markers and Molecules of Acute Pancreatitis - A Review
}

\author{
Vaishnavi Sundar ${ }^{1}$, Shalini Ramasamy ${ }^{2}$, Tiasha Dasgupta ${ }^{2}$, Sanjana Vimal ${ }^{1}$, Bagyashree VT ${ }^{1}$, Divya Raghunandan ${ }^{1}$, \\ Kshitija Joshi ${ }^{1}$, Venkatraman Manickam ${ }^{2}$, Ramasamy Tamizhselvi ${ }^{1 *}$ \\ ${ }^{1}$ Department of Biotechnology, School of Biosciences and Technology, Vellore Institute of Technology, Vellore, Tamil Nadu, India. \\ ${ }^{2}$ Department of Biomedical Sciences, School of Biosciences and Technology, Vellore Institute of Technology, Vellore, Tamil Nadu, India. \\ *Corresponding author's E-mail: tamizhselvi.r@vit.ac.in
}

Received: 20-05-2021; Revised: 24-07-2021; Accepted: 03-08-2021; Published on: 15-08-2021. \begin{abstract}
Acute pancreatitis (AP) being one of the rapidly emerging gastrointestinal concerns, needs early surveillance as the instantaneous inflammatory response in the pancreas may lead to severe pathological clinical phenotypes. Failure in monitoring AP may lead to severe inflammatory response syndrome (SIRS) which is seen amongst one-fifth of individuals requiring extensive management and palliative care to prevent the disease progression. However, the major question of defining a definitive pool of molecular targets for AP remains unanswered thus far. As a first step towards designing a definitive pool of molecular targets for AP, we aim to compile the reported evidence of potential signaling molecules in AP. During AP, the inflammatory response-inducing factors trigger multiple signaling molecules that cause the pancreatic tissue assault. As a counter-active response, the host body's immune system initiates the resolution of inflammation and repair of the damaged tissue by stimulating various anti-inflammatory signaling molecules. The communication between the components of these pro-inflammatory and anti-inflammatory signaling pathways opens up a wide window for the identification of various prognostic markers and molecular targets for the management of AP. This review collectively presents the available prognostic biomarkers in detail for a better prognosis of AP.
\end{abstract}

Keywords: AP, Redox signaling, Cytokines, SIRS, Apoptosis, Biomarkers.

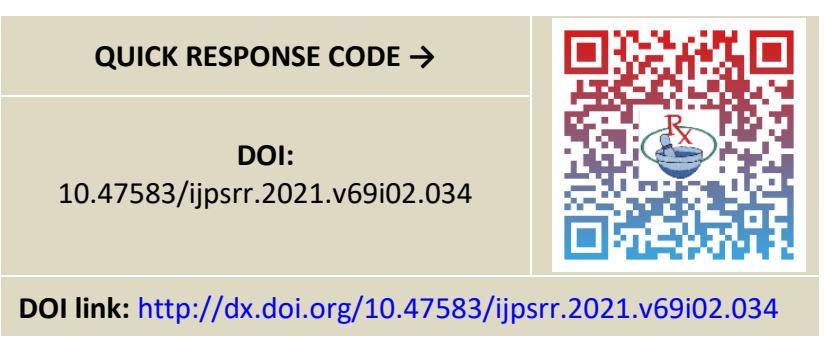

\section{INTRODUCTION}

A cell releases variety of signalling molecules that act as a ligand for the cell receptors facilitating communication during the physiological processes. These receptors can be intercellular or extracellular based on the type of trigger that elicit the particular response. In general, the chemical nature of a signalling molecule can be hydrophobic (that helps them to pass through the plasma membrane and bind to internal receptors like the steroid hormones) or hydrophilic (some proteins/peptides are water soluble and interact with the cell membrane receptors). The ligands bind to their respective receptors and initiate a cascade of chemical reactions known as a cell signalling pathway. (Fig.1). This cascade continues until the last signalling molecule gets activated and the desired physiological event occurs in the target cell ${ }^{1}$. Some of these signalling molecules act as prognostic or predictive markers during various disease conditions.

Prognostic markers are biomarkers used to measure the progress of a disease in the patient sample. Prognostic markers are useful to stratify the patients into groups, guiding towards precision medicine by providing collectively describing the overall disease outcome irrespective of treatment ${ }^{2,3}$.

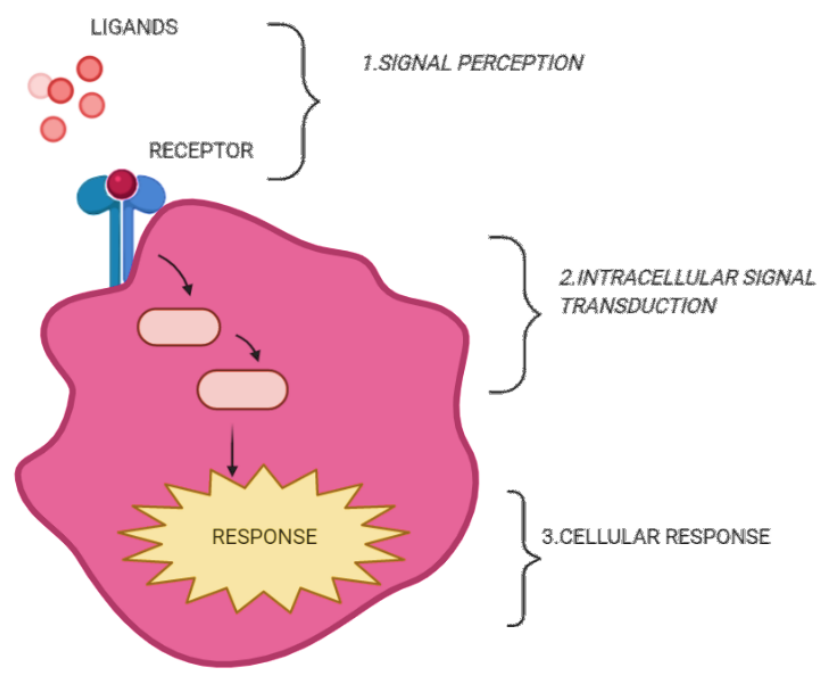

Figure 1: Illustration of Cell Signalling Process

On the other hand, a predictive biomarker gives information about the effect of a therapeutic intervention. A predictive biomarker can possibly become a potential therapeutic target against the disease in question. Some prognostic biomarkers of inflammation are Erythrocyte sedimentation rate (ESR), C-reactive protein (CRP) and plasma viscosity (PV) levels measured in blood ${ }^{2,4}$. Further, there are other types of molecules like biological gaseous mediators that can act as signalling molecules during 
inflammation ${ }^{5}$. For instance, the nitrous oxide (NO) can mediate the actions of macrophages and associated cytokines on the target cells ${ }^{6}$. In general, targeting these pathways is crucial for drug development as interception in these pathways majorly leaves an impact on disease progression and prognosis. Few examples of these pathways include pro-survival pathway (Protein kinase B /AKT signalling), Mitogen-activated protein kinase (MAPK) pathway, apoptosis signalling mechanisms, JAK-STAT signalling, Nf-kB Pathway, etc ${ }^{7}$. That being so, some of the potential prognostic and predictive markers of AP are discussed in this review. AP is an acute inflammatory condition of the pancreas resulting in release of digestive enzymes prematurely eliciting a severe immune response in the host. Several molecules and signalling pathways have been identified and targeted in AP pathogenesis so far.

\section{PATHOGENESIS OF AP}

Located within the retroperitoneum, the pancreas is well protected from both environmental and mechanical injury. AP is described as a cascade of inflammatory events involving sudden pancreatic autodigestion due to premature release of trypsin resulting from early zymogen activation. Patients can go on to develop mild to severe AP depending on the severity of tissue damage. It is widely understood that AP can result in multiple organ failure and systemic inflammatory response syndrome. In most cases necrotic foci are noted within the pancreatic tissue ${ }^{4-6}$. Severe AP can have several etiologies with gallstones and alcohol being the most prevalent ${ }^{3}$. In the majority of patients, the condition resolves without complications, however it can cause substantial complications and mortality in up to $25 \%$ of those needing hospitalization periods in an intensive care unit beyond 2 weeks ${ }^{8}$.

Impaired or hyperactive pancreas regeneration after injury would cause exocrine insufficiency or recurrent / chronic pancreatitis and potentially carcinogenesis. Given inflammation is a host immune response to the tissue injury, many immune cells are activated to restore the organ function and integrity. Macrophages are the most abundant immune cells in the regenerating pancreas; however, their phenotype and role remain poorly defined. Using caerulein-induced AP model it has been reported that $\mathrm{M} 1$ macrophages dominated the pro-inflammatory phase of AP, while M2-like macrophages dominated during pancreas repair/regeneration. Depletion of macrophages at early or late regenerative stage dramatically blocked the acinar-ductal metaplasia (ADM) or delayed inflammation resolution, respectively. Moreover, alternative activation of macrophages was partially dependent on IL-4RA signalling and ECM/AKT activation in pancreatic macrophages facilitated inflammation resolution during tissue regeneration ${ }^{9}$.

\section{CLINICAL MANIFESTATIONS AND PROGNOSIS OF AP}

Although most patients with AP have the mild form of the disease, about $20-30 \%$ develops a severe form, often associated with single or multiple organ dysfunction requiring intensive care. Early diagnosis for a severe case is one of the major challenges in managing severe $A P^{10}$. The assessment of the severity of AP is a critical early step in its management as it predicts prognosis. A range of options are available for this including clinical evaluation, standardized prognostic criteria, computed tomography (CT) and biochemical markers. Clinical assessment has limited predictive accuracy in case of AP. Therefore, additional assessment using biochemical and radiologic criteria in combination with standardized tests, is appropriate to determine severity and prognosis in AP. Although disease-specific therapy for AP remains limited at this point, accurate grading of severity can guide the clinician in directing severe cases to the intensive or specialty care and justifies more aggressive and potentially invasive management (e.g., endoscopic sphincterotomy, intravenous antibiotics) ${ }^{11}$.

The atherogenic index of plasma (AIP) reflects the levels of triglycerides (TG) and high-density lipoprotein (HDL) cholesterol. The AIP is a potential biomarker for the prediction of SAP in clinical practice. This result provides that impaired lipid metabolism is associated with the severity of pancreatitis.

Local and systemic lipotoxicity has been recognized as an important risk factor for SAP or multisystem of in AP. Evaluated the predictive ability of the AIP for the severity of AP compared with other scoring systems. Several tools have been developed for severity stratification in AP. They include single biochemical markers, imaging methods and complex scoring systems, all of which aim at an early detection of severe AP to optimise monitoring and treatment of patients as early as possible. Among single biochemical markers, $\mathrm{C}$-reactive protein (CRP) remains the most useful. Despite its delayed increase, peaking not earlier than $72 \mathrm{~h}$ after the onset of symptoms, it is accurate and widely available. Many other markers have been evaluated for their usefulness, and for some of them, very promising data could be shown. Among them interleukin 6 seems to be the most promising parameter for use in clinical routine. For the detection of pancreatic infection, procalcitonin is the most sensitive, and can be used as an indicator for the need for fine-needle aspiration of pancreatic necrosis.

Regarding imaging, contrast-enhanced computed tomography is still the reference method for the detection of necrotising AP. Pancreatitis-specific scoring systems have been shown to be of value for the prediction of severity and progression of AP, but cannot be applied any earlier than $48 \mathrm{~h}$ after admission to hospital. The APACHEIl score has not been developed specifically for AP and is rather complex to assess, but has been proven to be an early and reliable tool. Indication, timing and consequences of the methods applied need to be carefully considered and incorporated into clinical assessments to avoid costs and harm to the patient ${ }^{12}$. 


\section{SIGNALING PATHWAYS IN AP PATHOGENESIS}

The molecular signalling processes are complicated events stimulated by several families of signalling molecules inside the cells for cell growth, viability, differentiation, proliferation and various other physiological processes. A lot of diseases including inflammatory conditions, cancers and related autoimmune diseases occur due to faulty cell signalling events or due to an interruption in the signalling pathways ${ }^{13}$. Many types of cell communications are reported as the chemical mediators migrate to their appropriate target sites resulting in a cell response to the stimulus. Generally, the chemical mediators follow any of the following four signalling types namely: Paracrine signalling, autocrine signalling, endocrine signalling, and direct communication between neighbouring cells via gap junctions ${ }^{14}$.
Inflammation is a response of the body's immune system against tissue injury, this can be chronic or acute inflammation. Cytokines play a supreme role in the development of inflammation in pancreatic cells directed by the cytokine pathway which is regulated by cytokine signalling molecules and their receptors in the respective targeted cells. Cytokines are protein molecules secreted from cells to do the job of cell communication, they can act in the autocrine, paracrine and endocrine mode of cell signalling ${ }^{15}$. Pro-inflammatory and anti-inflammatory cytokines are two types of cytokine molecules based on their area of secretion- Th1 and Th2 cells and regulate the inflammatory reaction. In this section cytokine molecules which are significantly associated with AP will be discussed.

\section{INFLAMMATORY MEDIATORS AS PREDICTIVE MARKERS OF AP}

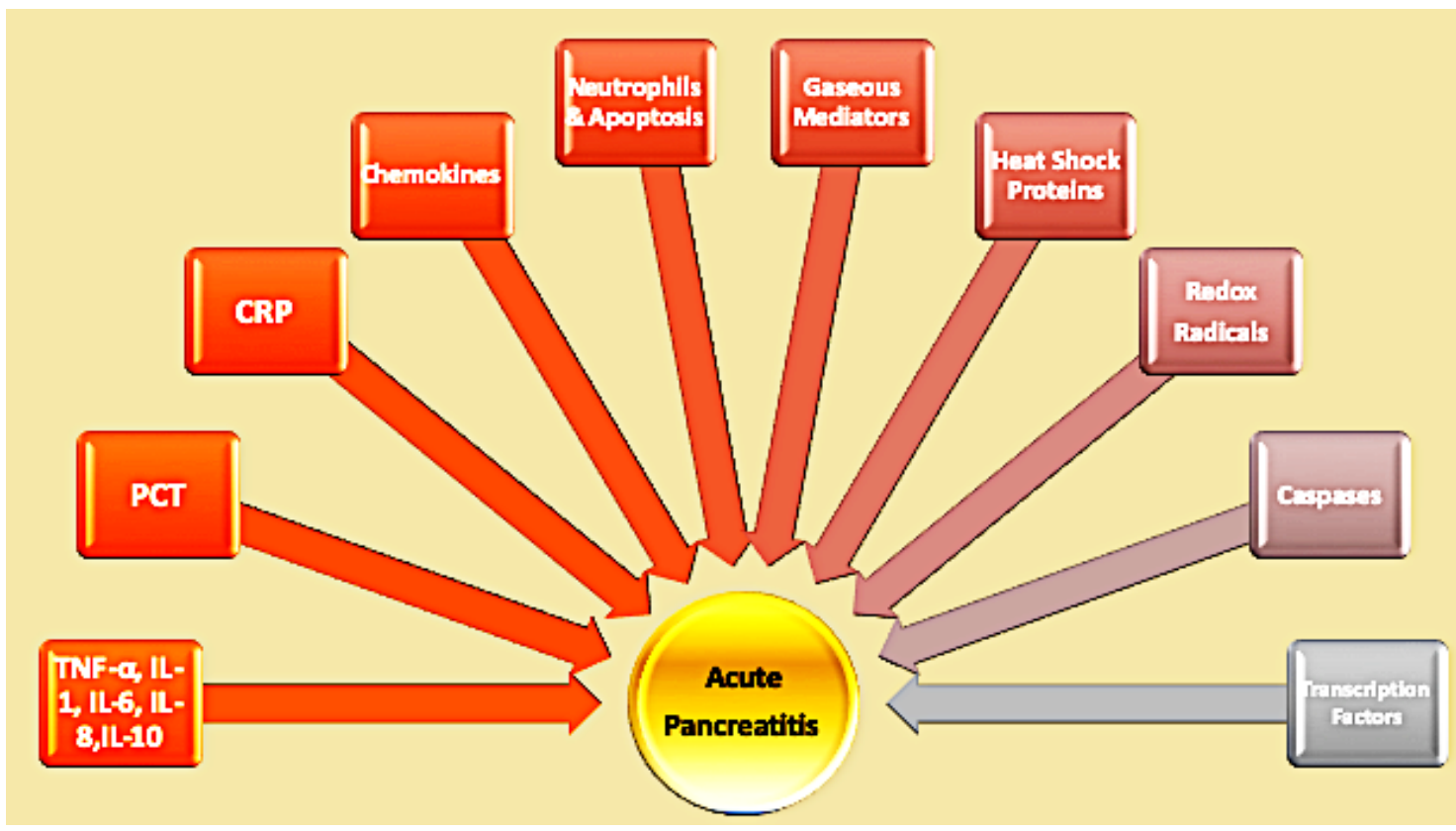

Figure 2: Inflammatory Mediators as Predictive markers for AP

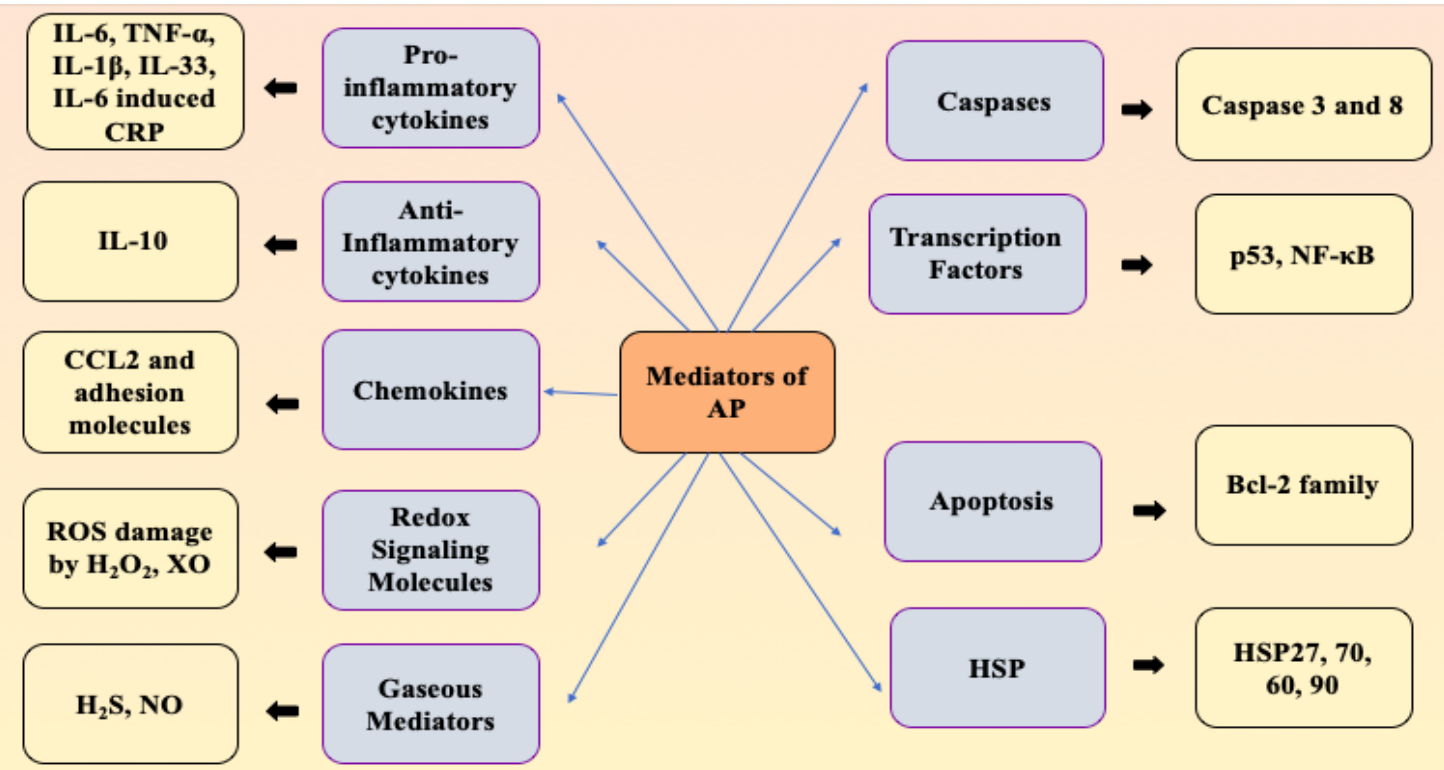

Figure 3: Types of mediators in AP disease progression 


\section{$5.1 \quad$ C-reactive protein (CRP)}

This is the most widely used predictor of severity in AP, though it is not specific. There is a $48-72$ hour delay until peak values are reached, which limits its use for early evaluation of the severity of the condition. At the time of admission to hospital, the sensitivity of CRP is $47 \%$ - which is similar to that of clinical examination. At 48 hours postsymptom onset, if a cut-off level of $150 \mathrm{mg} / \mathrm{l}$ is used, it has been shown to be an accurate severity predictor (sensitivity and specificity above $80 \%$ ). It is useful in the follow-up of the disease. The advantages of CRP are that it is easy to measure, cheap and widely available ${ }^{16}$.

\section{$5.2 \quad$ Interleukin-6}

The pro-inflammatory cytokine IL- 6 is the key inducer of CRP synthesis in the liver. Peak concentrations of IL- 6 are reached 24 to 48 hours before those of CRP. It distinguishes between severe and mild AP with $86 \%$ to $100 \%$ sensitivity and $71 \%$ to $100 \%$ specificity. The sensitivity of IL-6 for detecting severe AP was $100 \%$, with a specificity of $86 \%$ at a cut-off concentration of $2.7 \mathrm{ng} / \mathrm{L}$. Serum concentrations of IL- 6 decrease swiftly and its use in clinical practice has also been limited by the complexity and cost of the assay ${ }^{17}$. Moreover, IL- 6 works as a pro-inflammatory as well as antiinflammatory cytokine largely synthesized by lymphocytes and works on $B$ cells maturation to produce plasma cells to produce antibodies. It also decreases the action of proinflammatory cytokines like TNF-alpha and IL- $\beta^{18}$. On the other hand, it triggers the production of IL-10 which is a proinflammatory cytokine and helps tumour cells escape immune-surveillance ${ }^{19}$. The role of IL-6 in AP is major. Cerulein induced pancreatitis in mice models showed an increase in the amount of digestive enzyme production which led to acinar cell death followed by the production of excessive leukocytes which directly increase the production of pro-inflammatory cytokines like IL-6, TNF-alpha, IL-1beta. However, mice with an IL-6 knockout showed damage in pancreatic tissues suggesting its anti-inflammatory action. In AP, the release of IL- 6 and TNF-alpha leads to various secondary infections and an increased mortality ${ }^{20}$. A study has shown IL- 6 can be used as an early detection marker for AP patients in a case to study organ failure as the level of IL6 and IL- 8 are elevated compared to IL18 and TNF- $\alpha^{21}$.

\subsection{Procalcitonin (PCT)}

This is an acute phase reactant, considered to be one of the earliest and most specific markers of bacterial infections in immunocompromised and immunodeficient patients. Normally, the plasma PCT level in healthy individuals is very low $(<0.5 \mathrm{ng} / \mathrm{mL})$. It provides a rapid diagnostic test, available at the patient's bedside, and its half-life is suitable for daily monitoring of disease progress and treatment. It rises earlier than CRP in the course of severe infections and falls promptly with response to appropriate antibiotics. Post-operatively, PCT has proved to be a sensitive indicator of whether infected necrosis had been successfully evacuated, but surgery itself does not result in a postoperative PCT increase as long as infection is absent.
In contrast, acute phase parameters like CRP or IL-6 are elevated in response to both infectious and non-infectious (for example, post-operative) stimuli, so an estimation of the presence or absence of infection is not possible. In contrast, levels of IL- 6 were high in both infected and sterile pancreatic necrosis, and remained high for several days, even after surgical removal of the infected focus. Therefore, PCT is a readily available, helpful and an accurate marker that enables a decision concerning surgical therapy ${ }^{22}$.

\subsection{Interleukin-8 (IL-8)}

IL-8 level estimation is inferior in the prediction of infected necrosis when compared to PCT and CRP with an overall sensitivity of $72 \%$ in patients with infected necrosis. A constant rise in IL-8 concentrations to at least $140 \mathrm{pg} / \mathrm{mL}$, usually during the second week of the disease, is indicative of septic multiple organ failure but it is not reliable to PCT and even CRP level estimation ${ }^{21}$

\subsection{Tumor Necrosis Factor $-\alpha$ (TNF- $\alpha)$}

TNF- $\alpha$ is a research hotspot for many acute, chronic conditions involving inflammation, cancer as well as in autoimmune disorders ${ }^{23}$. TNF- $\alpha$ is a pro-inflammatory mediator that predominantly controls gene expression, cell viability etc. ${ }^{24}$. A large number of cells produces TNF-alpha but a major source of this molecule is Macrophages, this production is controlled by various factors, for example, nuclear factor kappa $B(N F-\kappa B)^{25}$. After binding of TNF molecule with its receptor, they initiate two different signaling complex, one which negatively controls antiapoptotic proteins and thus stop cell death and the other one initiates cell death after internalization receptors ${ }^{20,26}$.

Sodium taurocholate induced AP in rat model showed an increased level of TNF- $\alpha$ and other cytokines in serum ${ }^{27}$.

Studies showed AP increase the production of proinflammatory cytokines like TNF- $\alpha$ and IL-1 beta hereby increase damage in nearby organs as well as trigger acute lung injury ${ }^{28}$.

In Pancreatic Acinar Cells, TNF-alpha increases activation of protease before maturation and induce necrosis ${ }^{29}$. However, treating AP patients with somatostatin, ulinastatin and Saliva miltiorrhiza significantly decreased the level of TNF-alpha, IL6 on $4^{\text {th }}$ and $7^{\text {th }}$ day of treatment ${ }^{30}$.

\subsection{Interleukin -1 (IL-1)}

IL-1 is produced during acute and chronic inflammation, but showed no correlation with the severity of AP. Blockade of the cytokine cascade at the level of the IL-1 receptor before or soon after induction of pancreatitis significantly attenuates the rise in these cytokines and is associated with decreased severity of pancreatitis and reduced intrinsic pancreatic damage ${ }^{31}$. Thus, early prediction of the severity of AP can be made by well-validated scoring systems at 48 hours, but the serum markers procalcitonin and IL- 6 allow earlier prediction (12 to 24 hours after admission). 
IL-1 is a pro-inflammatory cytokine derived from macrophages majorly known for an innate immune response made up of 11 proteins and has a role in autoinflammatory diseases in humans ${ }^{32}$. They have seven agonist ligands molecules, one anti-inflammatory cytokine and three receptor antagonists. Ligands and receptors of IL1 family linked with chronic and acute inflammation ${ }^{33}$. IL 1 and TNF- alpha triggers the production of IL8 from monocytes, neutrophils and NK cells which has a role in AP. A study conducted with AP patient's serum sample on $1^{\text {st }}$, $3^{\text {rd }}$ and $5^{\text {th }}$ day of hospitalization showed an increased amount of IL-8 compare to control group ${ }^{34}$.

IL-1 has one more family member named IL-33 is also associated with inflammation, a study has been conducted where AP induced in mice and rats and performed ELISA for serum cytokines measurements where it was shown an increasing amount of IL-33 this study also showed that IL33 is associated with activation of acinar cell inflammatory pathways and cause inflammation in the pancreas ${ }^{35}$.

\subsection{Interleukin-10 (1L-10)}

Interleukin-10 acts as anti-inflammatory cytokine largely produced by macrophages and reduce the production of inflammatory mediators ${ }^{36}$. Irregular expression or dysregulation of IL-10 production is associated with inflammation and numerous auto-immune disease.

In mice model, sodium taurocholate induced AP was created and administrated to IL-10 in in vitro and in vivo. It was observed that IL-10 reduced inflammation in in vivo and reduce the action of TNF-alpha in in vitro ${ }^{37}$.

\subsection{Chemokines}

Chemokines are small protein molecules shares structural similarity with cytokines. [www.sciencedirect.com/topics/ medicine-and-dentistry/chemokine]. They work along with their receptors to control normal physiology, cell development and immune responses. Conserved position of cytokine residues divides chemokines into four groups which are $\mathrm{CXC}, \mathrm{CX} 3 \mathrm{C}$ or $\mathrm{C}$ and $\mathrm{CC}^{38}$. Studies have shown in AP excess amount of chemokine receptors are associated with acute lung injury whereas treatment with antagonist reduces the amount of receptor in case of $\mathrm{AP}^{39}$. One more study where AP was induced with cerulein showed an increasing amount of CCL2 chemokine receptor through Real-time PCR which suggests that Chemokines and their receptors can be used as an early marker ${ }^{40}$.

\subsection{Redox signaling molecules}

AP is a localised inflammation of the pancreas that must be considered together with conditions like sepsis, trauma etc that would culminate into systemic inflammatory response syndrome accompanied by multiple organ failure. Oxidative stress and redox signalling play an important role in the initiation and progression of the disease. The mechanisms involved in the progression of AP have not been clearly elucidated but it is well accepted that they start in the acinar cells which are the exocrine cells of the pancreas that produce and transport enzymes that are pass onto the duodenum where they will assist in the digestion of food and both alcohol consumption and gallstone play significant role in triggering AP.

The reactive oxygen species (ROS) cause a direct oxidative damage to the lipids and proteins that modulate redox sensitive transcription factors and signal-transduction pathways. ROS is an important player amongst others like pro-inflammatory cytokines, chemokines and platelet activation factors in the systemic progression of AP. Since the eighties, reports have suggested the use of antioxidants and pro-oxidants in the treatment of the disease, providing proof for the involvement of oxidative stress in AP. Recent studies have led to the conclusion that oxidative stress is not only a key mediator of the early events associated with AP but also of the associated systemic inflammatory response system ${ }^{41}$.

Hydrogen peroxidise $\left(\mathrm{H}_{2} \mathrm{O}_{2}\right)$ is a second messenger of the NADPH oxidases, a major source of ROS in inflammation that in its turn activates the NF- $\kappa \mathrm{B}$. The nuclear factorkappa $\mathrm{B}(\mathrm{NF}-\mathrm{KB})$ is a protein that specifically binds to the $\kappa \mathrm{B}$ site of cellular genes and induces transcription of the respective genes and is a key factor of the immune response, inflammation, stress, damage of tissues and apoptosis. Deletion of the $\kappa \mathrm{B}$ site from the NOS2 promoter will prevent the cytokine-induced increases in NOS2 transcription that regulates the activity of NF- $K B$.

In-situ studies in rats have shown that in mild AP due to overstimulation of cerulein, the free radical generation is prominently associated with the infiltration of activated neutrophils while on the other hand, in necrotic AP that is induced by taurocholate retrograde perfusion, the major event is the conversion of Xanthine Oxidase dehydrogenase $(\mathrm{XDH})$ to Xanthine Oxidase (XO), a pro-oxidant enzyme. Two different mechanisms account for the activation and conversion of $\mathrm{XDH}$ to $\mathrm{XO}$ in $\mathrm{AP}$ : activation of chymotrypsinogen to chymotrypsin may lead to activation of XDH to XO and oxidation of thiol groups could also lead to XO formation.

\section{$5.10 \quad$ Gaseous Mediators}

Hydrogen sulphide $\left(\mathrm{H}_{2} \mathrm{~S}\right)$ is a common endogenously produced gaseous signalling molecule, synthesized from Lcysteine primarily by two key enzymes:

\section{- $\quad$ cystathionine- $\gamma$-lyase (CSE) in the peripheral tissues}

- $\quad$ cystathionine- $\beta$-synthetase (CBS) in the central nervous system.

An increase in the levels of $\mathrm{H}_{2} \mathrm{~S}$ causes simultaneous increase in the levels of pancreatic cytokines and vice-versa and hence the levels of $\mathrm{H}_{2} \mathrm{~S}$ are correlated with disease severity $^{42}$. $\mathrm{H}_{2} \mathrm{~S}$ plays a dual role in AP based on its concentration in the system. It presents an antiinflammatory effect when present in low concentrations and cellular toxicity through the oxidative free radicals at high concentrations ${ }^{43}$. The anti-inflammatory effect is produced via the inhibition of the production of pro- 
inflammatory cytokines by activation of the phosphatidylinositol 3-kinase/protein kinase B pathway.

Nitric oxide (NO) is a highly reactive free radical produced by the action of $\mathrm{L}$-arginine through a series of isoenzymes known as nitric oxide synthase (NOS). NOS can be divided into three further categories, eNOS, iNOS, and nNOS. Amongst these, iNOS is expressed in a large number of cells ${ }^{44}$. The inducible nitric-oxide synthase (iNOS) contributes to large NO formation in inflammatory processes like AP. Although a normal level of NO is considered beneficial, uncontrolled production of $\mathrm{NO}$ is detrimental. Endogenous NO will protect against oxidative damage since inhibition of NOS with increase lipid peroxidation by $\mathrm{N}$-nitro-L-arginine methyl ester.

\subsection{Caspases and apoptosis in AP}

Apoptosis was initially used to define a morphologically distinct form of cell death (Kerr et al., 1972) but has since been understood to be a highly regulated and distinctive form of 'programmed' cell death involving biochemical events leading to changes in morphology and the subsequent elimination of cells. These morphological changes include the shrinking of cells, blebbing, nuclear and chromosomal fragmentation, DNA fragmentation etc. Apoptotic cell death in AP follows several different mechanisms and their study might offer important pharmacological insight ${ }^{45}$.

Among the protein families involved in apoptosis, the most prominent are Caspases. There are ten major caspases that have been categorized into initiators (caspase-2,-8,-9,-10), effectors (caspase-3,-6,-7) and inflammatory caspases (caspase-1,-4,-5) (Rai et al.,2005). Other caspases that have been identified are caspase 11,12, 13 and 14 which regulate apoptosis in specific situations and tissues. Caspases are activated by several pathogenic factors in AP. Substances like Cholecytokinin lead to the activation of caspase-8. It is an early regulator of the proteolytic cascade relating to apoptosis. Other reports suggest that factors like oxidative stress induce apoptosis of acinar cells in pancreatic cancer. This includes the activation of caspase-3 which in turn degrades DNA repair proteins Ku70 and Ku80.

\section{APOPTOSIS-ASSOCIATED GENE EXPRESSION IN AP.}

Another important functional component of the apoptotic pathway is the Bcl-2 family of regulators. This family includes BclxL, Bcl-w, A1/Bfl-1, Boo/ Diva, Mcl-1, Bax, Bak, $\mathrm{Bad}, \mathrm{Bcl} \times \mathrm{xLS}$, and other genes. Apoptosis is determined by the balance between the pro-apoptotic and anti-apoptotic genes of this family. Bcl-2 and BclxL inhibit apoptosis, however Bax can dimerize with them and inhibit their function, thereby promoting apoptosis. AP induces an increase in the levels of pancreatic Bax in acinar cells inducing apoptosis. Apart from the Bcl-2 family of genes, a stress-induced protein gene localized in acinar cells is overexpressed during pancreatitis leading to cell apoptosis.
6.1 Transcription Factor Involvement in Acinar Cell Apoptosis

The involvement of transcription factors in the signal transduction pathways steering apoptosis is still an area of scrutiny. The instances of the transcription factors highlighted below may serve to illustrate this statement. For instance, transcription factor p53. It is known that p53 induces bax expression, which further aids apoptotic initiation. However, it is unclear whether this transcription factor plays such a role in the pancreatic acinar cells as well. Research has shown that p53 may mediate apoptosis in AR4-2J cells following treatment with a higher than normal dose of cerulein. A report has even found pancreatic p53 mRNA levels to be temporarily coordinated with that of bax during AP. On the other hand, a different study found that while Bax expression was in synchrony with acinar cell apoptosis, there was no actual relationship with p53 itself.

Another example is that of the nuclear factor NF-KB which, along with activator protein-1, assists in the control of several immune and inflammatory response genes. There is reason to believe that NF-KB may also play a role in the initiation of the transduction pathways leading to apoptosis, even in pancreatic acinar cells. It has been hypothesized that NF-KB may induce cell death by activating TNF-alpha transcription. This has been further supported by research that found that NF-KB and activator protein-1 are activated by lyso-PC before apoptosis. Prior studies on lyso-PC have already suggested its connection to the pathogenesis of AP. However, further research must be conducted in order to truly implicate this factor in pancreatic apoptosis.

\subsection{Neutrophils and Apoptosis}

Neutrophil levels are an important indicator of the extent of progression of AP. Depletion of neutrophils were found to correspond with an increase in number of cells undergoing apoptosis (Sandoval et al.). Treatment with methotrexate also resulted in a significant decrease in number of neutrophils and a subsequent increase in cell death. Another study found that in a taurocholate-induced severe AP as the experimental model, neutrophils along with TNF alpha may be implicated in the apoptotic and necrotic forms of acinar cell death ${ }^{46}$.

Neutrophils and acinar cells release cytokines such as TNF$\alpha$ and interleukin-1 $\beta$ in order to induce inflammation. However, recent studies have intimated that these cytokines may be involved with the induction of pancreatic apoptosis as well. This is because acinar cells have been shown to release TNF- $\alpha$, which has been implicated in the progression to pancreatitis. Furthermore, TNF- $\alpha$ has been found to mediate apoptosis both in in vitro suspensions of acinar cells as well as in in vivo cerulein models of AP.

In addition, TNF- $\alpha$ has been shown to initiate acinar cell apoptosis in a time and dose dependent manner. It can also elicit mechanisms that both promote as well as inhibit apoptosis in the AR4-2J cells. This is due to the ability of 
TNF- $\alpha$ to activate TNFR, resulting in a balance between NF$\kappa B$ activation and the induction of the effectors of mitogenactivated protein kinases and Fas- associated death domain/ caspase activity. In several models using TNF- $\alpha$, mitochondrial permeability transition has been essential in releasing cytochrome $\mathrm{c}$ and further initiating apoptosis. It is already known that the opening of the mitochondrial permeability transition pore plays an important role in necrotic as well as apoptotic cell death, blurring the supposedly fundamental distinction between the two pathways.

As of now however, the apoptotic and necrotic mechanisms behind AP are still under inquiry. Both pathways however, involve several molecules that may be targeted for therapy. It is hence worthwhile to study and consider the selective induction of acinar apoptosis as an important factor in AP.

\section{HEAT SHOCK PROTEINS AS AP MODULATORS}

Cerulein as well as L-arginine-induced models of AP have been used to evaluate heat shock response. The relative expression level of HSP70 was found different in response to induction of pancreatitis and heat shock. Four isoforms of HSP70 were expressed in the pancreas in response to thermal stress in vivo. With induction of arginine pancreatitis there was increase in expression of HSP27, 70, 60 and 90. Lower dose of L-arginine, resulted in highest expression of HSP27 followed by HSP70, 60 and 90 . With a higher dose, HSP60 was overexpressed level followed by HSP27 and HSP70. HSP27 exists as three isoforms (unphosphorylated, mono- and diphosphorylated forms) in rat pancreatic acinar cells. HSP27 phosphorylation was stimulated by CCK in vivo and in vitro and by osmotic stress in vitro. Phosphorylation status of HSP27 was also altered with monophosphorylated form increasing at $6 \mathrm{~h}$ and remained elevated up to $120 \mathrm{~h}$. Dephosphorylated form was maximum at $12 \mathrm{~h}$ and decreased thereafter but was still higher compared to basal level. Injection of nonpathological dose of L-arginine $(3 \mathrm{~g} / \mathrm{kg})$ induced a higher ratio of phosphorylated to unphosphorylated HSP27 protein which indicates that it might be a protective response. Overexpression of HSP27 is known to confer resistance to heat and other stresses.

Some of the plausible mechanisms could be (i) decrease in cellular contents of ATP (ii) accumulation of unfolded proteins (iii) ischemia because of compromised microcirculation ${ }^{29}$. All of these are capable of triggering stress in the cell and a heat stress response. A severity assessment system consisting of 2 sets of factors - one is a prognostic score based on clinical parameters and the other based on contrast-enhanced computed tomography (CECT) imaging is developed recently. The prognostic score and CECT grade variables are shown in Tables 1 and 2 . If the patient is diagnosed with SAP by either prognostic score and/or CECT grade, the diagnosis of SAP is established. The prognostic score relies on the presence of base excess or shock, respiratory failure, age, SIRS criteria, elevated BUN, lactate dehydrogenase, CRP, and reduced platelet count and serum calcium. Having 3 or more abnormal variables indicates severe disease.

\section{CHALLENGES OF TARGETING SIGNALLING MOLECULES IN AP}

Despite hundreds of clinical trials, there is no licensed specific drug therapy for the disease. A recently published evidence-based review summarized the harsh reality that we have actually very little effective treatment modalities to date. The authors outlined many promising experimental and preliminary clinical studies that ultimately were proven to be ineffective in improving clinical outcomes. Nonetheless, progress has been made through reasonably reliable observations. Accurately predicting SAP has been a challenge for clinicians. There are a variety of scoring systems and prediction markers, including the Ranson criteria, Glasgow score, bedside index of severity in AP score (BISAP), APACHE II, systemic inflammatory response syndrome (SIRS), C-reactive protein (CRP), haematocrit, and blood urea nitrogen (BUN). Early severity assessment is important especially on the day of admission because this is considered the window of opportunity for application of interventions to prevent necrosis and organ failure.

A challenge for early prognostic systems is the difficulty in identifying patients who initially appear well, but subsequently deteriorate. Our ability to establish prognosis, especially in the earliest disease phases, for triaging patients or instituting specific therapies, still needs improvement. The paradigms used to predict the severity of AP are good, but only after several days of disease.

However, CECT fails sometimes to predict the formation of necrotizing pancreatitis, when taken very early after the onset. data suggest that perfusion CT might be an alternative measure to the clinical scores and CECT for risk stratification in SAP. It is clear that at present there are no reliable scoring systems or markers to predict either moderate or SAP and persistent organ failure. Further, the diagnosis of pancreatic or peripancreatic necrosis on CT scan, a good marker of severity, may require 72 hours after diagnosis to reach its peak. This period may be too late for a therapeutic intervention based on disease severity

It is commonly believed that AP evolves rapidly from an initial assault into local and systemic tissue damages. From there, the patient may develop a variety of complications or recover fully. Evidences suggest that current treatment modalities are gold standard of palliative care for AP but there is no complete cure for the disease still.

\section{CONCLUSION}

Unfortunately, there is no specific drug available to treat AP in early stages to prevent the moderate and severe forms. All the earlier RCTs with several pharmacological therapies, including glucagon, gabexate, somatostatin, and lexipafant failed to show a significant benefit. Hence, the current guidelines recommend only supportive care as the main treatment modality in AP. Even after the above mentioned negative trials, there have been several agents reported to 
be effective in experimental AP in recent years. However, bench to bedside translation of any of the agents has not happened and the main reason for this is the inherent difficulties in conducting drug intervention trial in AP. AP is almost always treated in the hospital setting, and thus different from diseases like inflammatory bowel disease (IBD) where the agents being tested are administered predominantly in the outpatient setting. There have been few trials of promising immunotherapeutic agents and no trials targeting primary acinar cell injury, even though there have been many preclinical studies of this last disease mechanism. There has therefore been a lack of translation. Other areas of medicine where drug discovery and development have been successful in bringing new therapies to the clinic are exemplars. Understanding of critical pathological mechanisms that can be targeted by drugs is important in the development of new treatments and underpins the uptake of new treatments.

One can find many current drug trials in AP from clinical trials.gov, but published positive studies include one using ulinastatin from India and another with pentoxifylline. Important requirements to overcome the above challenges are: (1) performing trials at institutions with significant number of patients with AP to avoid multicentre study which is difficult to conduct in AP due to logistics; 2) including an experienced principle investigator in the field of AP with a team; (3) establishing infrastructure for early identification of all patients admitted; (4) obtaining the resources to conduct the study. Early identification of patients with AP is important if we are to initiate studies within the first 24 hours after diagnosis of AP. From experimental and clinical experience, it appears that after the initial 24 to 72 hours, the inflammatory cascade may be fully established leading to multiorgan failure; this may limit the utility of any drug therapies ${ }^{47}$. A collaborative network with the overall goal of development of an effective, licensed therapy could enhance these endeavours.

\section{REFERENCES}

1. Navarro SL, Kantor ED, Song X, Milne GL, Lampe JW, Kratz M, et al. Factors associated with multiple biomarkers of systemic inflammation. Cancer Epidemiol Prev Biomarkers. 2016;25(3):52131 https://doi.org/10.1158/1055-9965.EPI-15-0956

2. Lapić I, Padoan A, Bozzato D, Plebani M. Erythrocyte sedimentation rate and C-reactive protein in acute inflammation: meta-analysis of diagnostic accuracy studies. Am J Clin Pathol. 2020;153(1):14-29. https://doi.org/10.1093/ajcp/aqz142

3. Corsonello A, Pedone C, Battaglia S, Paglino G, Bellia V, Incalzi RA. Creactive protein (CRP) and erythrocyte sedimentation rate (ESR) as inflammation markers in elderly patients with stable chronic obstructive pulmonary disease (COPD). Arch Gerontol Geriatr. 2011;53(2):190-5. https://doi.org/10.1016/j.archger.2010.10.015

4. Celiński K, Mądro A. Molecular mechanisms and biochemical markers of acute pancreatitis. Biochem Medica. 2008;18(2):175-82. https://doi.org/10.11613/BM.2008.017

5. Wallace JL, lanaro A, Flannigan KL, Cirino G. Gaseous mediators in resolution of inflammation. In: Seminars in immunology. Elsevier; 2015. p. 227-33. https://doi.org/10.1016/j.smim.2015.05.004

6. MacMicking J, Xie $Q$, Nathan $C$. Nitric oxide and macrophage function. Annu Rev Immunol. 1997;15(1):323-50. https://doi.org/10.1146/annurev.immunol.15.1.323
7. Crusz SM, Balkwill FR. Inflammation and cancer: Advances and new agents. Vol. 12, Nature Reviews Clinical Oncology. 2015. p. 584-96. https://doi.org/10.1038/nrclinonc.2015.105

8. Portelli $M$, Jones $C D$. Severe acute pancreatitis: pathogenesis, diagnosis and surgical management. Hepatobiliary Pancreat Dis Int. 2017;16(2):155-9. https://doi.org/10.1016/S1499-3872(16)60163-7

9. Wu J, Zhang L, Shi J, He R, Yang W, Habtezion A, et al. Macrophage phenotypic switch orchestrates the inflammation and repair/regeneration following acute pancreatitis injury. EBioMedicine. https://doi.org/10.1016/j.ebiom.2020.102920

10. Leppäniemi A, Tolonen M, Tarasconi A, Segovia-Lohse H, Gamberini E, Kirkpatrick AW, et al. 2019 WSES guidelines for the management of severe acute pancreatitis. World J Emerg Surg. 2019;14(1):1-20. https://doi.org/10.1186/s13017-019-0247-0

11. Triester SL, Kowdley K V. Prognostic factors in acute pancreatitis. J Clin Gastroenterol. 2002;34(2):167. https://doi.org/10.1097/00004836-200202000-00014

12. Schütte $\mathrm{K}$, Malfertheiner $\mathrm{P}$. Markers for predicting severity and progression of acute pancreatitis. Best Pract Res Clin Gastroenterol. 2008;22(1):75-90. https://doi.org/10.1016/j.bpg.2007.10.013

13. Meller A. A new tool for cell signalling research. Nat Nanotechnol. 2019;14(8):732-3. https://doi.org/10.1038/s41565-019-0505-z

14. Forms of Signaling. 2020.

15. Zhang J-M, An J. Cytokines, inflammation, and pain. Int Anesthesiol Clin. 2007:45(2):27-37. https://doi.org/10.1097/AlA.0b013e318034194e

16. Fisic E, Poropat $G$, Bilic-Zulle L, Licul V, Milic S, Stimac D. The role of IL-6, 8, and 10, sTNFr, CRP, and pancreatic elastase in the prediction of systemic complications in patients with acute pancreatitis. Gastroenterol Res Pract. 2013;2013. https://doi.org/10.1155/2013/282645

17. Viedma JA, Pérez-Mateo M, Domínguez JE, Carballo F. Role of interleukin-6 in acute pancreatitis. Comparison with C-reactive protein and phospholipase A. Gut. 1992 Sep;33(9):1264 LP - 1267. https://doi.org/10.1136/gut.33.9.1264

18. Spooren A, Kolmus K, Laureys G, Clinckers R, De Keyser J, Haegeman G, et al. Interleukin-6, a mental cytokine. Brain Res Rev. 2011;67(12):157-83. https://doi.org/10.1016/i.brainresrev.2011.01.002

19. Ara T, DeClerck YA. Interleukin- 6 in bone metastasis and cancer progression. Eur J Cancer. 2010;46(7):1223-31. https://doi.org/10.1016/j.ejca.2010.02.026

20. Shen $\mathrm{Y}, \mathrm{Cui} N$, Miao B, Zhao E. Immune dysregulation in patients with severe acute pancreatitis. Inflammation. 2011;34(1):36-42. https://doi.org/10.1007/s10753-010-9205-4

21. Malmstrøm ML, Hansen MB, Andersen AM, Ersbøll AK, Nielsen $\mathrm{OH}$, Jørgensen $\mathrm{LN}$, et al. Cytokines and organ failure in acute pancreatitis: inflammatory response in acute pancreatitis. Pancreas. 2012;41(2):271-7. https://doi.org/10.1097/MPA.0b013e3182240552

22. Rau B, Schilling MK, Beger HG. Laboratory markers of severe acute $\begin{array}{lll}\text { pancreatitis. Dig 2004;22(3):247-57. } & \end{array}$ https://doi.org/10.1159/000082796

23. Chu W-M. Tumor necrosis factor. Cancer Lett. 2013;328(2):222-5. https://doi.org/10.1016/j.canlet.2012.10.014

24. Park KM, Bowers WJ. Tumor necrosis factor-alpha mediated signaling in neuronal homeostasis and dysfunction. Cell Signal. 2010;22(7):977-83. https://doi.org/10.1016/j.cellsig.2010.01.010

25. Parameswaran $\mathrm{N}$, Patial $\mathrm{S}$. Tumor necrosis factor- $\alpha$ signaling in macrophages. Crit Rev Eukaryot Gene Expr. 2010;20(2). https://doi.org/10.1615/CritRevEukarGeneExpr.v20.i2.10

26. Cabal-Hierro L, Lazo PS. Signal transduction by tumor necrosis factor $\begin{array}{llll}\text { receptors. } & \text { Cell 2012;24(6):1297-305. }\end{array}$ https://doi.org/10.1016/i.cellsig.2012.02.006

27. Wang $Y$, Liu W, Liu X, Sheng M, Pei $Y$, Lei R, et al. Role of liver in 
modulating the release of inflammatory cytokines involved in lung and multiple organ dysfunction in severe acute pancreatitis. Cell Biochem Biophys. 2015;71(2):765-76. https://doi.org/10.1007/s12013-014-0261-5

28. Roberts NJ, Zhou S, Diaz Jr LA, Holdhoff M. Systemic use of tumor necrosis factor alpha as an anticancer agent. Oncotarget. 2011;2(10):739. https://doi.org/10.18632/oncotarget.344

29. Sendler M, Dummer A, Weiss FU, Krüger B, Wartmann $T$, Scharffetter-Kochanek K, et al. Tumour necrosis factor $\alpha$ secretion induces protease activation and acinar cell necrosis in acute experimental pancreatitis in mice. Gut. 2013;62(3):430-9. https://doi.org/10.1136/gutjnl-2011-300771

30. Wang G, Wen J, Wen P, Wilbur RR, Zhou S-F, Xiao X. The effect of somatostatin, ulinastatin and Salvia miltiorrhiza on severe acute pancreatitis treatment. Am J Med Sci. 2013;346(5):371-6. https://doi.org/10.1097/MAJ.0b013e31827aa2bc

31. Shao GN, Kim H, Imran SM. https://www. sciencedirect. com/science/article/abs/pii/S092633731500346X. 2016;

32. Weber A, Wasiliew P, Kracht M. Interleukin-1 (IL-1) Pathway. Sci Signal. $2010 \quad$ Jan;3(105):cm1 LP-cm1. https://doi.org/10.1126/scisignal.3105 cm1

33. Garlanda C, Dinarello CA, Mantovani A. The interleukin-1 family: back to the future. Immunity. 2013;39(6):1003-18. https://doi.org/10.1016/j.immuni.2013.11.010

34. Daniel P, Leśniowski B, Mokrowiecka A, Jasińska A, Pietruczuk M Małecka-Panas E. Circulating levels of visfatin, resistin and proinflammatory cytokine interleukin- 8 in acute pancreatitis. Pancreatology. https://doi.org/10.1159/000276986

35. Kempuraj D, Twait EC, Williard DE, Yuan Z, Meyerholz DK, Samuel I. The novel cytokine interleukin-33 activates acinar cell proinflammatory pathways and induces acute pancreatic inflammation in mice. PLoS One. 2013;8(2):e56866. https://doi.org/10.1371/journal.pone.0056866

36. Sabat R, Grütz G, Warszawska K, Kirsch S, Witte E, Wolk K, et al. Biology of interleukin-10. Cytokine Growth Factor Rev. 2010;21(5):331-44. https://doi.org/10.1016/j.cytogfr.2010.09.002

37. Lin $R$, Chen $F$, Wen $S$, Teng $T$, Pan $Y$, Huang $H$. Interleukin-10 attenuates impairment of the blood-brain barrier in a severe acute pancreatitis rat model. J Inflamm. 2018;15(1):1-12.

\section{https://doi.org/10.1186/s12950-018-0180-0}

38. Balkwill FR. The chemokine system and cancer. J Pathol. 2012;226(2):148-57. https://doi.org/10.1002/path.3029

39. Bhatia M, Zemans RL, Jeyaseelan S. Role of chemokines in the pathogenesis of acute lung injury. Am J Respir Cell Mol Biol. 2012;46(5):566-72. https://doi.org/10.1165/rcmb.2011-0392TR

40. Frossard JL, Lenglet S, Montecucco F, Steffens S, Galan K, Pelli G, et al. Role of CCL-2, CCR-2 and CCR-4 in cerulein-induced acute pancreatitis and pancreatitis-associated lung injury. J Clin Pathol. 2011;64(5):387-93. https://doi.org/10.1136/jcp.2010.088500

41. Pérez S, Pereda J, Sabater L, Sastre J. Redox Biology Redox signaling in acute pancreatitis. Redox Biol. 2015;5:1-14 https://doi.org/10.1016/j.redox.2015.01.014

42. Ji L, Li L, Qu F, Zhang G, Wang Y, Bai X, et al. Hydrogen sulphide exacerbates acute pancreatitis by over-activating autophagy via AMPK/mTOR pathway. J Cell Mol Med. 2016;20(12):2349-61. https://doi.org/10.1111/jcmm.12928

43. Wang G, Iv J-C, Wu L-F, Li L, Dong D-L, Sun B. From nitric oxide to hyperbaric oxygen: invisible and subtle but nonnegligible gaseous signaling molecules in acute pancreatitis. Pancreas. 2014;43(4):5117. https://doi.org/10.1097/MPA.0000000000000062

44. Xue M, Han L, Qian W, Li J, Qin T, Xiao Y, et al. Nitric Oxide Stimulates Acute Pancreatitis Pain via Activating the NF-KB Signaling Pathway and Inhibiting the Kappa Opioid Receptor. Oxid Med Cell Longev. 2020;2020. https://doi.org/10.1155/2020/9230958

45. Bhatia M, Fei LW, Cao $Y$, Hon $Y L$, Huang J, Puneet $P$, et al. Pathophysiology of acute pancreatitis. Pancreatology. 2005;5(23):132-44. https://doi.org/10.1159/000085265

46. Rau B, Paszkowski A, Esber S, Gansauge F, Poch B, Beger HG, et al. Anti-ICAM-1 antibody modulates late onset of acinar cell apoptosis and early necrosis in taurocholate-induced experimental acute pancreatitis. Pancreas. 2001;23(1):80-8. https://doi.org/10.1097/00006676-200107000-00012

47. Afghani E, Pandol SJ, Shimosegawa T, Sutton R, Wu BU, Vege SS, et al. Acute pancreatitis-progress and challenges: a report on an international symposium. Pancreas. 2015;44(8):1195. https://doi.org/10.1097/MPA.0000000000000500

Source of Support: The author(s) received no financial support for the research, authorship, and/or publication of this article.

Conflict of Interest: The author(s) declared no potential conflicts of interest with respect to the research, authorship, and/or publication of this article.

For any question relates to this article, please reach us at: editor@globalresearchonline.net New manuscripts for publication can be submitted at: submit@globalresearchonline.net and submit_ijpsrr@rediffmail.com 